\title{
Central nervous system metastasis secondary to colorectal cancer: a retrospective cohort study of 20 cases
}

\author{
Sebastián Mondaca', Valentina Hornig ${ }^{1}$, Pablo Munoz-Schuffenegger ${ }^{2,3,4}$, Francisco Acevedo ${ }^{1}$, Marcelo Garrido ${ }^{1}$ and Bruno Nervi ${ }^{1}$ \\ 1Departamento de Hematología-Oncología, Oncología Médica, Facultad de Medicina, Pontificia Universidad Católica de Chile, Diagonal Paraguay 319 , \\ Santiago, Chile 8330032 \\ ²Departamento de Hematología-Oncología, Radioterapia, Facultad de Medicina, Pontificia Universidad Católica de Chile, Diagonal Paraguay 319, \\ Santiago, Chile 8330032 \\ ${ }^{3}$ Department of Radiation Oncology, University of Toronto, Toronto, ON, Canada \\ ${ }^{4}$ Department of Radiation Oncology, Sunnybrook Health Sciences Centre, Odette Cancer Centre, Toronto, ON, Canada
}

Correspondence to: Sebastián Mondaca. E-mail: spmondac@uc.cl

\section{Abstract}

Introduction: Involvement of the central nervous system (CNS) secondary to colorectal cancer is infrequent and associated with a poor prognosis. Its treatment is extrapolated from metastases of other origins as the information available on this scenario is limited. The goal of this study is to assess the clinical characteristics of a series of patients and determine the results in terms of progression-free survival (PFS) and global survival.

Method: The records of patients with CNS metastasis of colorectal origin who were treated in this facility between the years 2001 and 2016 were reviewed retrospectively.

Results: 20 patients with CNS lesions of this origin were identified. Of these, $45 \%$ were male and $55 \%$ were female (average age 65.5 years). The histology corresponded to tubular adenocarcinoma in $95 \%$ of cases. Around $85 \%$ of the patients showed a neurological deficit, and their recursive partitioning analysis (RPA) classifications were 1 in $20 \%, 2$ in $55 \%$, and 3 in $25 \%$ of the cases studied. The treatments provided were: holocerebral radiotherapy (45\%), stereotactic radiosurgery $(25 \%)$, surgery followed by holocerebral radiotherapy $(25 \%)$, and exclusively palliative care (5\%). The PFS was 2.6 months from treatment of the CNS lesion, while the median survival was 3.8 months. The survival times for patients receiving different treatments were as follows: surgery plus holocerebral radiotherapy 16.2 months, stereotactic radiotherapy 12 months, and holocerebral radiotherapy 2.4 months $(p=0.003)$.

Conclusion: The prognosis for patients with metastasis of colorectal origin is poor. The patients treated with surgery or stereotactic radiotherapy can have a greater survival.

Keywords: Colorectal neoplasia, cerebral metastasis, radiosurgery, neurosurgery

Published: 21/12/2016

Received: 06/06/2016

ecancer 2016, 10:705 DOI: 10.3332/ecancer.2016.705

Copyright: (c) the authors; licensee ecancermedicalscience. This is an Open Access article distributed under the terms of the Creative Commons Attribution License (http://creativecommons.org/licenses/by/3.0), which permits unrestricted use, distribution, and reproduction in any medium, provided the original work is properly cited. 


\section{Introduction}

Colorectal cancer (CRC) is the third most common cancer and the fourth in mortality at the global level [1], and it is a public health issue for both developed countries and developing countries. Around $20 \%$ of patients show metastatic disease at the time of diagnosis and up to a third of patients may develop it, particularly in the liver [2]. The central nervous system (CNS) is an infrequent site of involvement, representing $1-4 \%$ of cases in different studies [3, 4], however, epidemiological data show an increasing trend in its occurrence [5, 6]. This can be explained further because of the success that has been achieved in managing extracranial metastatic illness. A recent systematic review reports that CRC is the principal cause of CNS metastasis of gastrointestinal origin representing 79\% of the total [7]. In patients with CRC, the presence of CNS involvement tends to be delayed and is associated with a poor prognosis with a median survival of about four months [8] which compares unfavorably with that of patients with CNS metastasis of other origin [9]. In the past few years, significant advances have been made in the field of neuroanesthesia, neurosurgical instruments, and techniques for stereotactic radiosurgery which have shown benefits in the treatment of patients with cerebral metastases overall [10, 11]. However, it has not been demonstrated that these treatments are effective in patients with CNS lesions of colorectal origin. In addition, the published studies are mainly of North American and Asian populations, and thus the possibility of extrapolating this data to other non-represented populations is limited.

The goal of this study is to retrospectively assess the clinical characteristics of a series of Latin American patients with CNS metastasis of colorectal origin and determine the results in terms of PFS and overall survival.

\section{Method}

A retrospective analysis of the database of patients treated with a diagnosis of CRC between the years 2001 and 2016 in the cancer centre of the Pontifica Universidad Católica de Chile was conducted. The diagnosis of CRC was reached through histological confirmation, and the clinical files of the patients were reviewed in order to extract the relevant clinical data. We used the American Joint Committee of Cancer (AJCC) TNM seventh edition classification for staging purposes. The diagnosis of CNS involvement was made using computed tomography (CT) with iodated contrast or magnetic resonance imaging (MRI) of the brain with gadolinium. The information on mortality was extracted from the Chilean Civil Registry Service database. The characteristics of the population were expressed as a mean for continual variables and as percentages for categorical variables. The statistical analysis was done using Kaplan-Meier curves with a log-rank test for the survival analysis. The programmes SPSS Version 21 and GraphPad Prism 7.0 were used to analyse the data.

Dispensing with consent was requested for this study and was approved by the institutional ethics committee of the Pontificia Universidad Católica de Chile.

\section{Results}

\section{Patients}

Of a total of 1154 patients with CRC who were treated during the time period of the study, we identified 20 patients with CNS metastasis of colorectal origin which corresponds to $1.7 \%$ of the total. Of these, $45 \%$ were male and $55 \%$ were female. The average age was 65.5 years (range between 42 and 84). The histological diagnosis corresponded to tubular adenocarcinoma in $95 \%$ of the patients, and $20 \%$ had CNS involvement at their initial consultation. The location of the primary tumour was in the colon in $70 \%$ of the cases (Table 1). The stage according to TNM at the time of diagnosis of the patients was Stage II: $10 \%$, Stage III: $35 \%$, and Stage IV: $55 \%$ of which $80 \%$ of the patients received chemotherapy prior to the diagnosis of metastatic involvement of the central nervous system, and a $50 \%$ of them also received biological medications such as bevacizumab, cetuximab, or panitumumab. 
A total of $65 \%$ of the patients showed a single metastasis at the time of diagnosis of intracranial illness. The location of the involvement of the CNS was supratentorial in $65 \%$ of the patients, infratentorial in $25 \%$, and the remaining $10 \%$ had involvement in both locations.

Among all $85 \%$ of the patients showed a neurological deficit, and their RPA classification [12] was 1 in $15 \%, 2$ in $65 \%$, and 3 in $25 \%$ of the patients (Table 2). Seven patients in the group were tested for the KRAS mutation which was present in one of them.

\section{Treatment and progression}

The average follow-up was 7.6 months. In this series, only $5 \%$ of the patients were treated exclusively with palliative care associated with the use of systemic steroids. A total of $65 \%$ were treated with holocerebral RT with a dose between 19 and 39 Gy which includes the suspension of treatment because of complications or clinical deterioration. Among all $25 \%$ were treated with stereotactic radiosurgery and $25 \%$ with surgery followed by holocerebral RT. Of the five patients treated with surgery, a complete resection (R0 resection) was achieved in four, while in one case residual microscopic illness was evident in the biopsy (R1 resection).

Table 1. Basal patient characteristics.

\begin{tabular}{|l|c|}
\hline & $\begin{array}{c}\text { Patients } \\
\text { (N = 20) }\end{array}$ \\
\hline Age (years) & 65.5 \\
\hline Sex (\%) & \\
Male & 45 \\
Female & 55 \\
\hline Location (\%) & \\
Colon & 70 \\
Rectum & 30 \\
\hline Histology (\%) & \\
Adenocarcinoma & 95 \\
Mucinous & 0 \\
Other & 5 \\
\hline AJCC Stage (\%) & \\
II & 10 \\
III & 35 \\
IV & 55 \\
\hline Tumour grade (\%) & \\
1 & 10 \\
2 & 80 \\
3 & 10 \\
\hline Prior Chemo (\%) & \\
Yes & 50 \\
No & 20 \\
\hline Prior biological & \\
treatment (\%) & 50 \\
Yes & \\
No & \\
\hline
\end{tabular}

Chemo: Chemotherapy 
Table 2. CNS involvement characteristics.

\begin{tabular}{|l|c|}
\hline & $\begin{array}{c}\text { Patients } \\
\text { (N = 20) }\end{array}$ \\
\hline Number of lesions (\%) & \\
Single & 65 \\
Multiple & 35 \\
\hline Location (\%) & \\
Supratentorial & 65 \\
Infratentorial & 25 \\
Both & 10 \\
\hline Lesion size (\%) & $\mathrm{N}=18$ \\
More than 3 cm & 28.6 \\
Less than or equal to 3 cm & 61.9 \\
\hline Time profile (\%) & \\
Synchronic & 20 \\
Metachronic & 80 \\
\hline Neurological deficit (\%) & \\
Yes & 85 \\
No & 15 \\
\hline RPA Classification (\%) & \\
1 & 20 \\
2 & 55 \\
3 & 25 \\
\hline Treatment (\%) & \\
Holocerebral RT & 25 \\
Stereotactic RT & \\
Surgery and holocerebral RT & \\
Palliative care & \\
\hline Posttreatment chemo (\%) & \\
Yes & \\
No & \\
\hline CNS: Central nervous system & \\
\hline
\end{tabular}

CNS: Central nervous system

RPA: Recursive partitioning analysis

RT: Radiotherapy

Chemo: Chemotherapy

The PFS was 2.6 months from treatment of the CNS lesion, while the overall survival (OS) was 3.8 months. Survival at one year was $20 \%$, and at two years was $5 \%$ (Figure 1). The subgroups treated with stereotactic radiosurgery or with surgery plus holocerebral RT had a survival of 12 and 16.2 months respectively. The subgroup treated only with holocerebral RT had a median survival of 2.4 months $p=0.003$ (Figure 2), while the patient treated with palliative care had a survival of 3.6 months. The OS according to RPA was 5.5 months for one, 3.8 months for two, and 3.6 months for three, $p=0.2$ (Figure 2). Of the patients, $35 \%$ received systemic chemotherapy following treatment of the intracranial illness.

\section{Discussion}

Our retrospective study of 20 patients with CNS involvement of colorectal origin confirms a poor prognosis for this group with a median survival of 3.8 months. One patient had a survival of more than two years which supports the use of aggressive treatment based on surgery 
or stereotactic radiosurgery. Most modern studies showed a similar survival compared to ours of about five months [7, 13, 14], although other studies have reported a better survival of nearly ten months [15]. The survival of patients treated with stereotactic radiosurgery or surgery followed by holocerebral RT was markedly greater than that of patients treated only with holocerebral RT. This finding can be explained by the selection bias associated with the fact that patients with factors indicating a poor prognosis were treated with holocerebral RT. Regarding this point, there are studies in which multivariant analysis validates the type of treatment as a factor in the prognosis [15, 16], while others have indicated the opposite [17, 18]. RPA is a validated prognostic classification which is often used in clinical practice which considers the factors related to the general condition of the patient and the control of systemic extracranial disease [12, 19]. Our study did not show significant variation in survival according to RPA classification. Another issue that should be noted is that of the high level of exclusive infratentorial involvement which was observed in a quarter of the patients. This has been described in other studies too where they have observed the presence of infratentorial lesions in between 22 and $55 \%$ of the patients with CRC and CNS metastasis [20]. A relevant limitation of our study is the relatively small number of patients, which makes it impossible to perform a multivariant analysis of prognostic factors. In addition, the data was collected retrospectively, so there was not a strict protocol for the diagnosis or treatment of these patients. For this reason there is a significant selection bias for the various treatments which prevents us from reaching definitive conclusions on their effectiveness. Among the strengths of this study is the fact that the treatment was provided in a single academic centre which has a tumour registry for the identification of patients. To our knowledge, this is the first study on Latin American patients with CNS involvement of colorectal origin. This has a relevance by becoming the inclusion data which represents different populations.

The prognosis for patients with metastatic $\mathrm{CRC}$ has significantly improved in the past few years, however, there is still no clinically relevant molecular classification. In this sense we believe that the model of patients with CNS metastasis could be interesting for the development of biomarkers and understanding the mechanisms of metastasis, as has been suggested by other research groups [21].

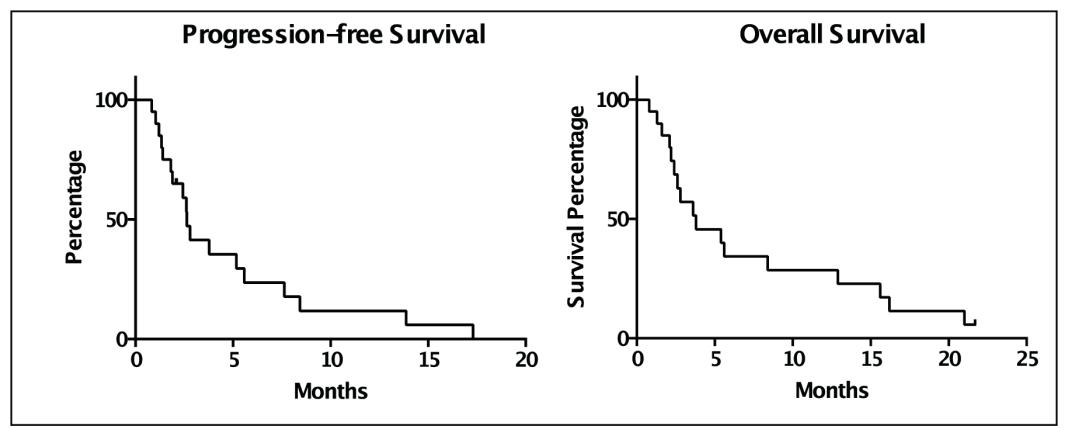

Figure 1. Kaplan Meier Curves for the assessment of progression-free survival and overall survival.

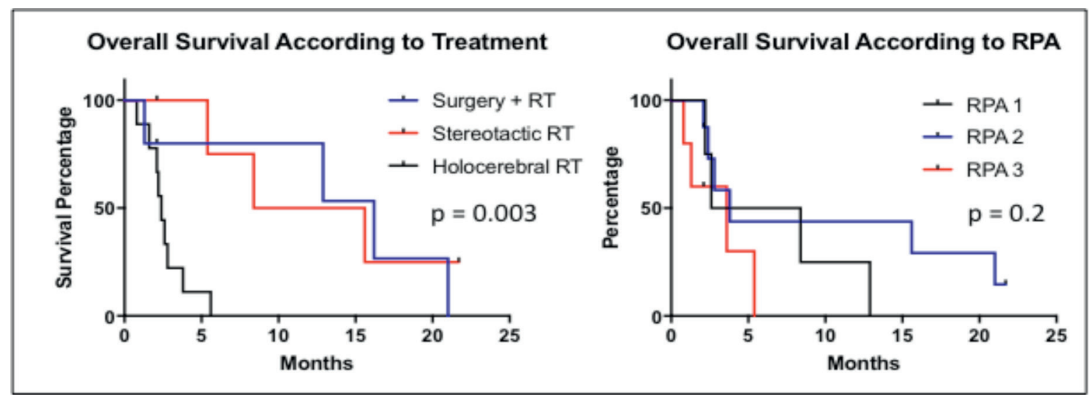

Figure 2. Kaplan Meier Curves for the assessment of progression-free survival and overall survival according to treatment and RPA. 


\section{Conclusion}

In conclusion, our study supports the poor prognosis of the patients with CNS involvement of colorectal origin and suggests that patients treated in an aggressive manner with surgery followed by holocerebral RT or stereotactic radiosurgery can have a longer survival even exceeding two years.

\section{References}

1. Ferlay J et al GLOBOCAN 2008- cancer incidence and mortality worldwide: IARC CancerBase No 10. Lyon: International Agency for Research on Cancer http://globocan.iarc.fr2010

2. Leporrier $\mathrm{J}$ et al (2006) A population-based study of the incidence, management and prognosis of hepatic metastases from colorectal cancer Br J Surg 93(4) 465-74 DOI: 10.1002/bjs.5278 PMID: 16523446

3. Cascino TL et al (1983) Brain metastases from colon cancer J Neurooncol 1(3) 203-9 DOI: 10.1007/BF00165604 PMID: $\underline{6678969}$

4. Jung $\mathrm{M}$ et al (2011) Brain metastases from colorectal carcinoma: prognostic factors and outcome $J$ Neurooncol 101(1) 49-55 DOI: $10.1007 / \mathrm{s} 11060-010-0214-9$

5. Nieder $\mathrm{C}$ et al (2011) Presentation, patterns of care, and survival in patients with brain metastases: what has changed in the last 20 years? Cancer 117(11) 2505-12 DOI: 10.1002/cncr.25707 PMID: 24048799

6. Smedby KE et al (2009) Brain metastases admissions in Sweden between 1987 and 2006 Br J Cancer 101(11)1919-24 DOI: 10.1038/sj.bjc.6605373 PMID: 19826419 PMCID: 2788258

7. Esmaeilzadeh $\mathrm{M}$ et al (2014) Brain metastasis from gastrointestinal cancers: a systematic review Int J Clin Pract 68(7) 890-9 DOI: $10.1111 /$ ijcp.12395 PMID: 24666726

8. Jung $\mathrm{YH}$ et al (2011) An experience of cyberknife treatment in patients with advanced pancreaticobilliary malignancy Korean J Gastroenterol 58(5) 264-9 DOI: 10.4166/kjg.2011.58.5.264 PMID: 22113043

9. Go PH et al (2011) Gastrointestinal cancer and brain metastasis: a rare and ominous sign Cancer 117(16) 3630-40 DOI: 10.1002/cncr.25940 PMID: 21319152

10. Kalkanis SN et al (2010) The role of surgical resection in the management of newly diagnosed brain metastases: a systematic review and evidence-based clinical practice guideline J Neurooncol 96(1) 33-43 DOI: 10.1007/s11060-009-0061-8 PMCID: $\underline{2808516}$

11. Bhangoo SS et al (2011) Evidence-based guidelines for the management of brain metastases Neurosurg Clin N Am 22(1) 97-104 DOI: $10.1016 /$ j.nec.2010.09.001

12. Gaspar L et al (1997) Recursive partitioning analysis (RPA) of prognostic factors in three Radiation Therapy Oncology Group (RTOG) brain metastases trials Int J Radiat Oncol Biol Phys 37(4) 745-51 DOI: 10.1016/S0360-3016(96)00619-0 PMID: 9128946

13. Kye BH et al (2012) Brain metastases from colorectal cancer: the role of surgical resection in selected patients Colorectal Dis 14(7) e378-85 DOI: 10.1111/j.1463-1318.2012.02962.x PMID: 22288509

14. Magni E et al (2014) Brain metastases from colorectal cancer: main clinical factors conditioning outcome Int $J$ Colorectal Dis 29(2) 201-8 DOI: 10.1007/s00384-013-1781-y

15. Gu XD et al (2015) Prognostic factors and multidisciplinary treatment modalities for brain metastases from colorectal cancer: analysis of 93 patients BMC Cancer 15902 DOI: 10.1186/s12885-015-1933-2 PMID: 26572484 PMCID: $\underline{4647298}$ 
16. Sun Z et al (2016) Clinical features and prognostic factors of brain metastasis from colorectal cancer Zhonghua Zhong Liu Za Zhi 38(1) 63-8 PMID: 26796809

17. Noura S et al (2012) Brain metastasis from colorectal cancer: prognostic factors and survival J Surg Oncol 106(2)144-8 DOI: 10.1002/jso.23055 PMID: $\underline{22287384}$

18. Bartelt S (2004) Patients with brain metastases from gastrointestinal tract cancer treated with whole brain radiation therapy: prognostic factors and survival World J Gastroenterol 10(22) 3345-8 DOI: 10.3748/wjg.v10.i22.3345 PMID: 15484315 PMCID: $\underline{4572310}$

19. Gaspar LE et al (2000) Validation of the RTOG recursive partitioning analysis (RPA) classification for brain metastases Int $J$ Radiat Oncol Biol Phys 47(4) 1001-6 DOI: 10.1016/S0360-3016(00)00547-2 PMID: 10863071

20. Mongan JP et al (2009) Brain metastases from colorectal cancer: risk factors, incidence, and the possible role of chemokines Clin Colorectal Cancer 8(2) 100-5 DOI: 10.3816/CCC.2009.n.016 PMID: 19739271 PMCID: 3909497

21. Zang YW et al (2012) Brain metastases from colorectal cancer: microenvironment and molecular mechanisms Int J Mol Sci 13(12) 15784-800 DOI: 10.3390/ijms131215784 